\title{
Keystroke Biometric System Using Wavelets
}

\author{
Woojin Chang \\ Department of Industrial Engineering, Seoul National University, \\ San 56-1, Sillim-dong, Gwanak-gu, Seoul 151-742, Korea \\ changw@snu.ac.kr
}

\begin{abstract}
We developed the keystroke biometric system (KBS) using the statistical features of the discrete wavelet transformed keystroke pattern in the frequency domain in addition to those of the original keystroke pattern in the time domain. Only 20 keystroke patterns of user's password typing, where the length of password is no more than 10, are used for building a KBS. The features in the time domain and those in the frequency domain are separately scored by the rules that we developed, and arbitrary given keystroke patterns are classified on the basis of total scores. The results show that our KBS is competitive in comparison with others due to its cheap computational cost, cheap usability cost, and the practically acceptable classification accuracy.
\end{abstract}

Keywords: Keystroke Dynamics, Keystroke Biometric System, Keystroke Authentication, Discrete Wavelet Transform.

\section{Introduction}

Keystroke biometric system (KBS) authenticates the legitimate user by his or her keystroke dynamics. KBS classifies an arbitrary keystroke typing pattern as either the legitimate user's or imposter's, and has two types errors for false acceptance and false rejection. The false acceptance rate (FAR) is the percentage of imposters' keystroke typing patterns identified as the genuine user's, and the false rejection rate (FRR) is the percentage of legitimate user's keystroke typing patterns identified as the imposters'. From the nature of two errors, FAR can be reduced at the cost of FRR, and vice versa.

One possible way to achieve high classification accuracy, cheap computational cost and cheap usability cost together is to use the statistical features of the transformed keystroke patterns in the frequency domain in addition to those of the original keystroke patterns in the time domain. The novelty of our research is that the statistical features of keystroke dynamics are directly detected and measured not only in the time domain, but also in the frequency domain by simple and basic statistical method that we developed. Furthermore, we use only 20 keystroke patterns of user's password typing to build a KBS.

\section{Keystroke Timing Vector and Wavelet Transformation}

A keystroke pattern is expressed as a keystroke timing vector (KTV) consisting of the sequences of duration and interval time pairs measured at the accuracy of 
milliseconds(ms). In the timing vector, each keystroke duration time is followed by the interval time which is calculated by subtracting the key-hit time from the previous key-release time. Thus, the interval time can be a negative value when a key is stroked before a previous key is released. This research includes the "Enter" keystroke as the last element of KTV. In this set-up, typing a string of $n$ characters results in a KTV of length $2 n+1$, which consists of $n+1$ keystroke duration times including the "Enter" key and $n$ keystroke interval times.

Discrete wavelet transformation (DWT) is applied to a KTV in the time domain, and then the corresponding keystroke wavelet coefficient vector (KWV) is produced in the frequency domain. Since DWT separates a KTV into multiresolution components, the latent features in KTV can be well observed and extracted in the KWV. Since the adequate data size for DWT should be 2 to the power of any natural number, the dummy data points need to be padded to the unfit KTV. The resulting modified vector $\mathbf{y}$ for DWT is as follows,

$$
\mathbf{y}=\left(y_{1}, \ldots, y_{2^{m}}\right)=(\underbrace{0, \ldots, 0}_{\left\lceil\frac{2^{m}-N}{2}\right\rceil}, v_{1}, \ldots, v_{N}, \underbrace{0, \ldots, 0}_{\left\lfloor\frac{2^{m}-N}{2}\right\rfloor}) .
$$

For a KTV of length $N, \mathbf{v}=\left(v_{1}, \ldots, v_{N}\right)$, the smallest $2^{m}$ larger than $N$ $\left(2^{m-1}<N \leq 2^{m}\right)$ is the adequate vector length for DWT. In this research, two zero vectors of length $\left\lceil\frac{2^{m}-N}{2}\right\rceil$ ( where $\lceil x\rceil$ is the smallest integer larger than or equal to $x$ ) and $\left\lfloor\frac{2^{m}-N}{2}\right\rfloor$ ( where $\lfloor x\rfloor$ is the largest integer less than or equal to $x$ ) need to be put into the front side and the back side of the KTV respectively. The piecewise constant function $f$ on $[0,1)$ generated by $\mathbf{y}$ can be represented as the corresponding wavelet decomposition as $f(x)=\sum_{k=0}^{2^{m}-1} y_{k} \cdot \mathbf{1}\left\{k 2^{-m} \leq\right.$ $\left.x<(k+1) 2^{-m}\right\}=c_{00} \phi(x)+\sum_{j=1}^{m-1} \sum_{k=0}^{2^{j}-1} d_{j k} \psi_{j k}(x)$, where $\mathbf{1}\{A\}$ is 1 if the condition ' $A$ ' is satisfied and 0 otherwise, $\phi(x)=\mathbf{1}\{0 \leq x<1\}$ is the scaling function, and $\psi_{j k}(x)=2^{\frac{j}{2}} \psi\left(2^{j} x-k\right)$ is a dilation and translation of Haar wavelet, $\psi(x)=\mathbf{1}\left\{0 \leq x<\frac{1}{2}\right\}-\mathbf{1}\left\{\frac{1}{2} \leq x<1\right\}$. Note the set $\left\{\psi_{j k}, j \in \mathbb{Z}, k \in \mathbb{Z}\right\}$ defines an orthogonal basis of $L^{2}(\mathbb{R})[1$.

The wavelet coefficients, $c_{00}$ and $d_{j k}$ 's are obtained from $y_{k}$ 's in the following way. When we let $\mathbf{c}_{m}=\left(c_{m 0}, \ldots, c_{m 2^{m}-1}\right)$ denote $\mathbf{y}$, and define $\mathbf{c}_{j}=$ $\left(c_{j 0}, \ldots, c_{j 2^{j}-1}\right)$ and $\mathbf{d}_{j}=\left(d_{j 0}, \ldots, d_{j 2^{j}-1}\right)$ for $j=1, \ldots, m-1, \mathbf{c}_{j}$ and $\mathbf{d}_{j}$ are calculated as follows. $c_{m-1 \ell}=c_{m 2 \ell} / \sqrt{2}+c_{m 2 \ell+1} / \sqrt{2}, \quad d_{m-1 \ell}=c_{m 2 \ell} / \sqrt{2}-$ $c_{m 2 \ell+1} / \sqrt{2}$, where $\ell=0, \ldots, 2^{m-1}-1$. For each $j=1, \ldots, m-1, c_{j-1 \ell}=$ $c_{j 2 \ell} / \sqrt{2}+c_{j 2 \ell+1} / \sqrt{2}, d_{j-1 \ell}=d_{j 2 \ell} / \sqrt{2}-d_{j 2 \ell+1} / \sqrt{2}$, where $\ell=0, \ldots, 2^{j-1}-1$. Then the wavelet coefficients, $\mathbf{w}=\left(\mathbf{d}_{m-1}, \mathbf{d}_{m-2}, \ldots, \mathbf{d}_{1}, d_{00}, c_{00}\right)$ can be obtained through cascade algorithm whose computational complexity is $O\left(2^{m}\right)$. w is the keystroke wavelet coefficient vector $(\mathrm{KWV})$ corresponding to $\mathbf{v}$.

Let $\#_{0}(\cdot)$ denote the number of 0 's in a vector. It can be shown that $\#_{0}(\mathbf{w})=$ $\sum_{j=1}^{m-2}\left\lfloor\left\lceil\frac{2^{m}-N}{2}\right\rceil \cdot 2^{-j}\right\rfloor+\sum_{j=1}^{m-2}\left\lfloor\left\lfloor\frac{2^{m}-N}{2}\right\rfloor \cdot 2^{-j}\right\rfloor<\#_{0}(\mathbf{y})=2^{m}-N$. This shows that the number of nonzero elements in $\mathbf{w}$ is larger than the number in $\mathbf{y}$. This implies that through DWT the information contained in $N$ elements of $\mathbf{v}$ can be diffused into more than $N$ elements of $\mathbf{w}$ in the frequency domain. By eliminating the zero elements from $\mathbf{w}$ and retaining nonzero elements of $\mathbf{w}$, the reduced 
KWV, $\mathbf{u}$ of length $\left(2^{m}-\#_{0}(\mathbf{w})\right)$ is constructed. The overall DWT process in this section can be illustrated as $\mathbf{v} \rightarrow \mathbf{y} \rightarrow \mathbf{w} \rightarrow \mathbf{u}$, and $\operatorname{dim}(\mathbf{v})<\operatorname{dim}(\mathbf{u})$, where $\operatorname{dim}(\cdot)$ is the length of a vector dimension.

\section{Authentication of Keystroke Dynamics}

In this paper, we measure how much different the given KTV is from the average user's pattern, and determine whether it belongs to user's or imposter's. Thus, the first step of KBS design should be the construction of user's keystroke dynamics reference consisting of the statistical characteristics of the elements of user's KTV and KWV.

Since the elements having a consistent pattern compose user's keystroke dynamics features, the KTV or KWV element having high consistency need to be selected for user's keystroke dynamics reference. This selection process results in the dimension reduction effect of KTV and KWV.

Let $\boldsymbol{\nu}$ and $\boldsymbol{\omega}$ denote user's sample KTV and the corresponding KWV, respectively. The consistency level at each element of user's KTV and KWV can be estimated from $\boldsymbol{\nu}$ 's and $\boldsymbol{\omega}$ 's, respectively. Assume that $M \mathrm{KTVs}, \boldsymbol{\nu}_{i}=$ $\left(\nu_{i 1}, \ldots, \nu_{i N}\right) i=1, \ldots, M$, are collected from the user. The corresponding $\mathrm{KWV}, \boldsymbol{\omega}_{i}=\left(\omega_{i 1}, \ldots, \omega_{i 2^{m}}\right)$ is obtained from $\boldsymbol{\nu}_{i}$, and the zero elements in $\boldsymbol{\omega}_{i}$ deleted, so that $\boldsymbol{\omega}_{i}$ is reduced to become $\boldsymbol{\mu}_{i}=\left(\mu_{i 1}, \ldots, \mu_{i 2^{m}}-\#_{0}\left(\boldsymbol{\omega}_{i}\right)\right)$. The consistency at the $j$ th element of $\boldsymbol{\nu}$ and $\boldsymbol{\mu}$ are represented as $p_{j}$ and $q_{j}$ respectively, as follows. After letting $p_{1}=q_{1}=1$, for $j=2, \ldots, N$,

$$
p_{j}=1 / M \cdot \max \left(\sum_{i=1}^{M} \mathbf{1}\left\{\operatorname{sgn}\left(\nu_{i j}-\nu_{i j-1}\right)=1\right\}, \sum_{i=1}^{M} \mathbf{1}\left\{\operatorname{sgn}\left(\nu_{i j}-\nu_{i j-1}\right)=-1\right\}\right),
$$

and for $j=2, \ldots, 2^{m}-\#_{0}(\boldsymbol{\omega})$,

$$
q_{j}=1 / M \cdot \max \left(\sum_{i=1}^{M} \mathbf{1}\left\{\operatorname{sgn}\left(\mu_{i j}-\mu_{i j-1}\right)=1\right\}, \sum_{i=1}^{M} \mathbf{1}\left\{\operatorname{sgn}\left(\mu_{i j}-\mu_{i j-1}\right)=-1\right\}\right),
$$

where $\operatorname{sgn}(x)=\mathbf{1}\{x>0\}-\mathbf{1}\{x<0\}$.

To construct the keystroke dynamics reference comprising the vector elements with high consistency level only, the following thresholding rule is applied to $\boldsymbol{\nu}_{i}$ and $\boldsymbol{\mu}_{i}(i=1, \ldots, M)$.

$$
\begin{aligned}
\tilde{\nu}_{i j} & =\nu_{i j} \cdot \mathbf{1}\left\{p_{j}>T\right\} j=1, \ldots, N \\
\tilde{\mu}_{i j} & =\mu_{i j} \cdot \mathbf{1}\left\{q_{j}>T\right\} j=1, \ldots, 2^{m}-\# o\left(\boldsymbol{\omega}_{i}\right)
\end{aligned}
$$

where $T(0 \leq T<1)$ is the threshold. By eliminating the zero elements from $\tilde{\boldsymbol{\nu}}_{i}=\left(\tilde{\nu}_{i 1}, \ldots, \tilde{\nu}_{i N}\right)$ and $\tilde{\boldsymbol{\mu}}_{i}=\left(\tilde{\mu}_{i 1}, \ldots, \tilde{\mu}_{i 2^{m}-\# o\left(\boldsymbol{\omega}_{i}\right)}\right)$ and retaining nonzero elements, the reduced vector $\boldsymbol{\nu}_{i}^{*}=\left(\nu_{i 1}^{*}, \ldots, \nu_{i R}^{*}\right)$ and $\boldsymbol{\mu}_{i}^{*}=\left(\mu_{i 1}^{*}, \ldots, \mu_{i S}^{*}\right)$ are constructed. In comparisons with $\boldsymbol{\nu}_{i}$ and $\boldsymbol{\mu}_{i}, \boldsymbol{\nu}_{i}^{*}$ and $\boldsymbol{\mu}_{i}^{*}$ are reduced in size and their overall patterns become more consistent. 
After these processes, we obtain user's keystroke dynamics reference consisting of the sample averages, $\overline{\boldsymbol{\nu}}^{*}=\left(\bar{\nu}_{1}^{*}, \ldots, \bar{\nu}_{R}^{*}\right)$ and $\overline{\boldsymbol{\mu}}^{*}=\left(\bar{\mu}_{1}^{*}, \ldots, \bar{\mu}_{R}^{*}\right)$, and the corresponding sample standard deviations, $\mathbf{s}_{\nu^{*}}=\left(s_{\nu_{1}^{*}}, \ldots, s_{\nu_{R}^{*}}\right)$ and $\mathbf{s}_{\mu^{*}}=\left(s_{\mu_{1}^{*}}, \ldots, s_{\mu_{S}^{*}}\right)$. By using these sample statistics $\overline{\boldsymbol{\nu}}^{*}, \overline{\boldsymbol{\mu}}^{*}, \mathbf{s}_{\nu^{*}}$, and $\mathbf{s}_{\mu^{*}}$ in user's keystroke dynamics reference, the difference between any given keystroke pattern and user's average keystroke pattern is measured.

Let $\mathbf{v}$ denote an arbitrary KTV, which is either the user's or the imposter's, and $\mathbf{w}$ denote the corresponding KWV. To classify $\mathbf{v}$ on the basis of the statistical features of keystroke dynamics defined in the framework of user's keystroke dynamics reference, $\mathbf{v}$ and $\mathbf{w}$ are processed in the same way as $\boldsymbol{\nu}$ and $\boldsymbol{\mu}$. By eliminating zero elements and retaining nonzero elements in $\mathbf{w}$, the reduced $\mathrm{KWV}$, $\mathbf{u}$ is obtained. In $\mathbf{v}$ and $\mathbf{u}$, the $j$ th element is kept if the corresponding $j$ th element in $\boldsymbol{\nu}$ and $\boldsymbol{\mu}$ is used for user's keystroke dynamics reference, and eliminated otherwise. Then, $\mathbf{v}^{*}=\left(v_{1}^{*}, \ldots, v_{R}^{*}\right)$ and $\mathbf{u}^{*}=\left(u_{1}^{*}, \ldots, u_{S}^{*}\right)$, which correspond to $\boldsymbol{\nu}^{*}$ and $\boldsymbol{\mu}^{*}$ are constructed. Note that $\operatorname{dim}\left(\mathbf{v}^{*}\right)=R \leq \operatorname{dim}(\mathbf{v})=N$ and $\operatorname{dim}\left(\mathbf{u}^{*}\right)=S \leq \operatorname{dim}(\mathbf{u})=2^{m}-\# o(\mathbf{w})$.

Using the elements of $\mathbf{v}^{*}$ and $\mathbf{u}^{*}$, the features of $\mathbf{v}$ are defined in the following three ways: size of vector element $\left(v_{j}^{*}\right.$ for $j=1, \ldots, R$, and $u_{j}^{*}$ for $j=1, \ldots, S$ ), $\operatorname{sign}$ of vector element $\left(\operatorname{sgn}\left(v_{j}^{*}\right)\right.$ for $j=1, \ldots, R$, and $\operatorname{sgn}\left(u_{j}^{*}\right)$ for $\left.j=1, \ldots, S\right)$, and sign of the difference between two values of vector elements $\left(\operatorname{sgn}\left(v_{j}^{*}-v_{\ell}^{*}\right)\right.$ for $j=$ $1, \ldots, R-1, \ell=j+1, \ldots, R, \operatorname{sgn}\left(u_{j}^{*}-u_{\ell}^{*}\right)$ for $\left.j=1, \ldots, S-1, \ell=j+1, \ldots, S\right)$.

An arbitrary keystroke pattern is scored by the appropriate rule incorporating the statistical keystroke dynamics features. For the convenient descriptions of the rules, the early defined $\boldsymbol{\nu}^{*}, \boldsymbol{\mu}^{*}, \mathbf{v}^{*}$, and $\mathbf{u}^{*}$ are used from this point on. Before the description of each rule, the followings need to be mentioned. First, $\alpha_{n}$ 's and $\beta_{n}$ 's are the constant values giving weight to the scoring sources of $v_{j}^{*}$ 's in the time domain and to those of $u_{j}^{*}$ 's in the frequency domain, respectively. Second, the scoring sources of $v_{j}^{*}$ and $u_{j}^{*}$ are multiplied by $p_{j}$ and $q_{j}$ respectively, to have weight.

- Rule 1: Measure the sizes of $v_{j}^{*}$ and $u_{j}^{*}$, and give penalty for $v_{j}^{*}$ and $u_{j}^{*}$ with abnormal size.

$$
\begin{aligned}
& \operatorname{score}_{1}^{\alpha}\left(\mathbf{v}^{*}\right)=\sum_{j=1}^{R}\left(\alpha_{1} p_{j} \mathbf{1}\left\{\bar{\nu}_{j}^{*} \neq 0\right\} \frac{\left|v_{j}^{*}-\bar{\nu}_{j}^{*}\right|}{s_{\nu_{j}^{*}}}+\sum_{k=2}^{5} \alpha_{k} p_{j} \mathbf{1}\left\{\left|v_{j}^{*}-\bar{\nu}_{j}^{*}\right|>k s_{\nu_{j}^{*}}\right\}\right) \\
& \operatorname{score}_{1}^{\beta}\left(\mathbf{u}^{*}\right)=\sum_{j=1}^{S}\left(\beta_{1} q_{j} \mathbf{1}\left\{\bar{\mu}_{j}^{*} \neq 0\right\} \frac{\left|u_{j}^{*}-\bar{\mu}_{j}^{*}\right|}{s_{\mu_{j}^{*}}}+\sum_{k=2}^{5} \beta_{k} q_{j} \mathbf{1}\left\{\left|u_{j}^{*}-\bar{\mu}_{j}^{*}\right|>k s_{\mu_{j}^{*}}\right\}\right)
\end{aligned}
$$

Note $\bar{\nu}_{j}^{*}, \bar{\mu}_{j}^{*}$ are the sample means, and $s_{\nu_{j}^{*}}, s_{\mu_{j}^{*}}$ are the sample standard deviations.

- Rule 2: Give penalty when $\operatorname{sgn}\left(v_{j}^{*}\right) \neq \operatorname{sgn}\left(\bar{\nu}_{j}^{*}\right)$ or $\operatorname{sgn}\left(u_{j}^{*}\right) \neq \operatorname{sgn}\left(\bar{\mu}_{j}^{*}\right)$.

$\operatorname{score}_{2}^{\alpha}\left(\mathbf{v}^{*}\right)=\sum_{j=1}^{R} \alpha_{6} p_{j}\left|\operatorname{sgn}\left(v_{j}^{*}\right)-\operatorname{sgn}\left(\bar{\nu}_{j}^{*}\right)\right|, \operatorname{score}_{2}^{\beta}\left(\mathbf{u}^{*}\right)=\sum_{j=1}^{S} \beta_{6} q_{j}\left|\operatorname{sgn}\left(u_{j}^{*}\right)-\operatorname{sgn}\left(\bar{\mu}_{j}^{*}\right)\right|$ 
- Rule 3: Give penalty when $\operatorname{sgn}\left(v_{j}^{*}-v_{j+1}^{*}\right) \neq \operatorname{sgn}\left(\bar{\nu}_{j}^{*}-\bar{\nu}_{j+1}^{*}\right)$ or $\operatorname{sgn}\left(u_{j}^{*}-\right.$ $\left.u_{j+1}^{*}\right) \neq \operatorname{sgn}\left(\bar{\mu}_{j}^{*}-\bar{\mu}_{j+1}^{*}\right)$.

$$
\begin{aligned}
\operatorname{score}_{3}^{\alpha}\left(\mathbf{v}^{*}\right) & =\sum_{j=1}^{R-1} \alpha_{7} p_{j+1} \mathbf{1}\left\{\operatorname{sgn}\left(v_{j}^{*}-v_{j+1}^{*}\right) \neq \operatorname{sgn}\left(\bar{\nu}_{j}^{*}-\bar{\nu}_{j+1}^{*}\right)\right\} \frac{\left|v_{j+1}^{*}-\bar{\nu}_{j+1}^{*}\right|}{s_{\nu_{j+1}^{*}}} \\
\operatorname{score}_{3}^{\beta}\left(\mathbf{u}^{*}\right) & =\sum_{j=1}^{S-1} \beta_{7} q_{j+1} \mathbf{1}\left\{\operatorname{sgn}\left(u_{j}^{*}-u_{j+1}^{*}\right) \neq \operatorname{sgn}\left(\bar{\mu}_{j}^{*}-\bar{\mu}_{j+1}^{*}\right)\right\} \frac{\left|u_{j+1}^{*}-\bar{\mu}_{j+1}^{*}\right|}{s_{\mu_{j+1}^{*}}}
\end{aligned}
$$

- Rule 4: Give penalty when $\operatorname{sgn}\left(v_{j}^{*}-v_{k}^{*}\right) \neq \overline{\operatorname{sgn}\left(\nu_{j}^{*}-\nu_{k}^{*}\right)}=\sum_{i=1}^{M} \operatorname{sgn}\left(\nu_{i j}^{*}-\right.$ $\left.\nu_{i k}^{*}\right) / M$ and $\operatorname{sgn}\left(u_{j}^{*}-u_{k}^{*}\right) \neq \overline{\operatorname{sgn}\left(\mu_{j}^{*}-\mu_{k}^{*}\right)}=\sum_{i=1}^{M} \operatorname{sgn}\left(\mu_{i j}^{*}-\mu_{i k}^{*}\right) / M$.

$$
\begin{aligned}
\operatorname{score}_{4}^{\alpha}\left(\mathbf{v}^{*}\right) & =\sum_{j=1}^{R-1} \sum_{k=j+1}^{R} \alpha_{8} p_{j} p_{k}\left|\operatorname{sgn}\left(v_{j}^{*}-v_{k}^{*}\right)-\overline{\operatorname{sgn}\left(\nu_{j}^{*}-\nu_{k}^{*}\right)}\right| \\
\operatorname{score}_{4}^{\beta}\left(\mathbf{u}^{*}\right) & =\sum_{j=1}^{S-1} \sum_{k=j+1}^{S} \beta_{8} q_{j} q_{k}\left|\operatorname{sgn}\left(u_{j}^{*}-u_{k}^{*}\right)-\overline{\operatorname{sgn}\left(\mu_{j}^{*}-\mu_{k}^{*}\right)}\right|
\end{aligned}
$$

Combining the above four rules, the total score for $\mathbf{v}$ is made as $\operatorname{score}(\mathbf{v})=$ $\sum_{n=1}^{4}\left(\operatorname{score}_{n}^{\alpha}\left(\mathbf{v}^{*}\right)+\operatorname{score}_{n}^{\beta}\left(\mathbf{u}^{*}\right)\right)$ We also define score ${ }^{\alpha}\left(\mathbf{v}^{*}\right)=\sum_{n=1}^{4} \operatorname{score}_{n}^{\alpha}\left(\mathbf{v}^{*}\right)$ as the scoring function for $\mathbf{v}^{*}$ in the time domain, and score ${ }^{\beta}\left(\mathbf{u}^{*}\right)=\sum_{n=1}^{4} \operatorname{score}_{n}^{\beta}\left(\mathbf{u}^{*}\right)$ as the scoring function for $\mathbf{u}^{*}$ in the frequency domain. To discriminate imposter's keystroke patterns from user's, the distribution of user's keystroke dynamics scores is obtained by the calculation of $\operatorname{score}\left(\boldsymbol{\nu}_{i}\right)$ for $i=1, \ldots, M$. The truncated sample score mean $\left(\overline{\mathrm{score}}_{95}\right)$ and the truncated sample standard deviation $\left(s_{95}(\right.$ score $\left.)\right)$ are calculated after excluding highest $5 \%$ of score $\left(\boldsymbol{\nu}_{i}\right) \mathrm{s}$. An arbitrary $\mathbf{v}$ is classified as the user's if score $(\mathbf{v}) \leq \overline{\text { score }}_{95}+t \cdot s_{95}$ (score), and $\mathbf{v}$ is classified as imposter's otherwise. Note that $t$ value relates to user's security setting, and the small $t$ can result in low FAR and high FRR, and the large $t$ can result in high FAR and low FRR. When score ${ }^{\alpha}\left(\mathbf{v}^{*}\right)$ or score ${ }^{\beta}\left(\mathbf{u}^{*}\right)$ is used only as the scoring function for $\mathbf{v}$, the same classification rule as in $\operatorname{score}(\mathbf{v})$ is applied.

\section{Experimental Results}

For the evaluation of the KBS using wavelets, the data set from Yu and Cho 3 . were used in this paper. In this research, to construct user's keystroke dynamics reference, 20 keystroke patterns were randomly selected from user's training data set. Thus, only 20 keystroke patterns $(M=20)$ were provided to build a KBS. For the evaluation of the KBS, two test sets of 75 keystroke patterns, one from the user and the other from the imposters were used. Thresholding with $T=0.8$ was applied to the corresponding KTVs and KWVs.

Table 1 shows the test results for the keystroke dynamics of 21 passwords typing, the dimensions of $\mathbf{v}, \mathbf{v}^{*}, \mathbf{u}^{*}$, and the FARs and FRRs of the classifications 
using score ${ }^{\alpha}\left(\mathbf{v}^{*}\right), \operatorname{score}^{\alpha}\left(\mathbf{u}^{*}\right)$, and score $(\mathbf{v}) \cdot \operatorname{score}^{\alpha}\left(\mathbf{v}^{*}\right), \operatorname{score}^{\beta}\left(\mathbf{u}^{*}\right)$, and score $(\mathbf{v})$ are evaluated in terms of accuracy by using the data mentioned above. The values of $\alpha_{n}$ and $\beta_{n}$ for $n=1, \ldots, 8$ were determined using 20 user's keystroke patterns $\left(\boldsymbol{\nu}_{i}, i=1, \ldots, 20\right)$ in the heuristic way that prioritizes the scoring sources of $v_{j}^{*}$ 's and $u_{j}^{*}$ 's and makes the user's keystroke dynamics scores, $\operatorname{score}\left(\boldsymbol{\nu}_{i}\right) i=1, \ldots, 20$ around 100 .

Table 1. The test results for keystroke dynamics of 21 passwords typing, the dimensions of $\mathbf{v}, \mathbf{v}^{*}, \mathbf{u}^{*}$, and the FARs and FRRs of the classifications using $\operatorname{score}^{\alpha}\left(\mathbf{v}^{*}\right)$, score $^{\alpha}\left(\mathbf{u}^{*}\right)$, and score $(\mathbf{v})$. Note 'c.s.93/ksy 8' contains special characters.

\begin{tabular}{|c|c|c|c|c|c|c|c|c|c|}
\hline \multirow{2}{*}{$\begin{array}{l}\text { User's } \\
\text { Password }\end{array}$} & \multirow[b]{2}{*}{$\operatorname{dim}(\mathbf{v})$} & \multirow[b]{2}{*}{$\operatorname{dim}\left(\mathbf{v}^{*}\right)$} & \multirow[b]{2}{*}{$\operatorname{dim}\left(\mathbf{u}^{*}\right)$} & \multicolumn{2}{|c|}{ score $^{\alpha}\left(\mathbf{v}^{*}\right)$} & \multicolumn{2}{|c|}{ score $^{\beta}\left(\mathbf{u}^{*}\right)$} & \multicolumn{2}{|c|}{ score $(\mathbf{v})$} \\
\hline & & & & FAR & $\overline{F R R}$ & FAR & $\overline{\text { FRR }}$ & FAR & FRR \\
\hline loveis. & 15 & 12 & 9 & 0 & 9.33 & 4.00 & 5.33 & 0 & 5.33 \\
\hline i love 3 & 17 & 14 & 12 & 6.67 & 1.33 & 6.67 & 0 & 5.33 & 0 \\
\hline 90200jdg & 17 & 7 & 11 & 2.67 & 16.00 & 1.33 & 16.00 & 0 & 16.00 \\
\hline autumnman & 19 & 15 & 16 & 0 & 4.00 & 0 & 8.00 & 0 & 4.00 \\
\hline tjddmswjd & 19 & 16 & 17 & 0 & 14.67 & 0 & 16.00 & 0 & 12.00 \\
\hline dhfpql. & 15 & 11 & 7 & 24.00 & 4.00 & 0 & 10.67 & 2.67 & 5.33 \\
\hline love wjd & 17 & 10 & 15 & 10.67 & 9.33 & 2.67 & 12.00 & 2.67 & 10.67 \\
\hline ahrfus8 & 17 & 11 & 15 & 21.33 & 4.00 & 4.00 & 5.33 & 0 & 5.33 \\
\hline dusru427 & 17 & 17 & 14 & 0 & 9.33 & 0 & 1.33 & 0 & 2.67 \\
\hline manseii & 17 & 11 & 11 & 6.67 & 12.00 & 4.00 & 10.67 & 5.33 & 12.00 \\
\hline drizzle & 15 & 11 & 10 & 0 & 2.67 & 2.67 & 1.33 & 0 & 2.67 \\
\hline beaupowe & 17 & 10 & 11 & 2.67 & 2.67 & 0 & 2.67 & 0 & 1.33 \\
\hline tmdwnsl1 & 17 & 15 & 15 & 2.67 & 4.00 & 0 & 13.33 & 0 & 10.67 \\
\hline yuhwa1kk & 17 & 15 & 17 & 0 & 0 & 0 & 0 & 0 & 0 \\
\hline anehwksu & 17 & 16 & 12 & 10.67 & 1.33 & 1.33 & 6.67 & 1.33 & 2.673 \\
\hline rhkdwo & 13 & 13 & 8 & 0 & 6.67 & 20.00 & 4.00 & 2.67 & 5.33 \\
\hline rla sua & 17 & 15 & 16 & 0 & 1.33 & 0 & 2.67 & 0 & 1.33 \\
\hline dlfjs wp & 17 & 15 & 18 & 1.33 & 2.67 & 1.33 & 1.33 & 0 & 2.67 \\
\hline dltjdgml & 17 & 16 & 16 & 0 & 0 & 0 & 2.67 & 0 & 1.33 \\
\hline dirdhfmw & 17 & 11 & 17 & 6.67 & 2.67 & 4.00 & 5.33 & 2.67 & 6.67 \\
\hline c.s.93/ksy 8 & 21 & 18 & 21 & 1.33 & 4.00 & 0 & 6.67 & 0 & 4.00 \\
\hline Minimum & & & & 0 & 0 & 0 & 0 & 0 & 0 \\
\hline Maximum & & & & 24.00 & 16.00 & 20.00 & 16.00 & 5.33 & 16.00 \\
\hline Average & & & & 4.64 & 5.40 & 2.48 & 6.29 & 1.08 & 5.33 \\
\hline
\end{tabular}

Note: For score ${ }^{\alpha}\left(\mathbf{v}^{*}\right)$ calculation, we used $\alpha_{1}=\alpha_{2}=1, \alpha_{3}=\alpha_{4}=\alpha_{5}=10, \alpha_{6}=$ $1, \alpha_{7}=2.5, \alpha_{8}=1.9$ and $\beta_{k}=0$ for $k=1, \ldots, 8$. For score ${ }^{\alpha}\left(\mathbf{u}^{*}\right)$ calculation, we used $\alpha_{k}=0$ for $k=1, \ldots, 8$ and $\beta_{1}=\beta_{2}=1, \beta_{3}=\beta_{4}=\beta_{5}=10, \beta_{6}=1, \beta_{7}=$ 2.5, $\beta_{8}=1$.9. For score $(\mathbf{v})$ calculation, we used $\alpha_{1}=\alpha_{2}=\beta_{1}=\beta_{2}=1, \alpha_{3}=\alpha_{4}=$ $\alpha_{5}=\beta_{3}=\beta_{4}=\beta_{5}=10, \alpha_{6}=\beta_{6}=1, \alpha_{7}=\beta_{7}=2.5, \alpha_{8}=\beta_{8}=1.9$. We used $t=\frac{\overline{\text { score }}_{95}}{s_{95}(\text { score })} \cdot \mathbf{1}\left\{\frac{\overline{s c o r e}_{95}}{s_{95}(\text { score })}<4.45\right\}+4.45 \cdot \mathbf{1}\left\{\frac{\overline{\text { score }}_{95}}{\left.\text { s.5 }_{95} \text { (score }\right)} \geq 4.45\right\}$ empirically. 
From the table, it can be said that $\operatorname{score}(\mathbf{v})$ performs best overall, and score ${ }^{\beta}$ $\left(\mathbf{u}^{*}\right)$ does better than score ${ }^{\alpha}\left(\mathbf{v}^{*}\right)$ in that FAR has priority over FRR when the difference between average FRRs is small. This implies that the distinct features of keystroke dynamics tend to be better expressed by using wavelet transformed keystroke patterns in the frequency domain than original keystroke patterns in the time domain, and the classification incorporating both the statistical features in the time and frequency domains are more effective than the classification incorporating those either in the time domain or in the frequency domain only.

\section{Conclusions}

The nonzero FARs in table 1 indicate the need for the improvement of classification accuracy. However, in the practical view, the KBS using score(v) is quite competitive due to the following reasons. First, the computational cost is very cheap since the complexity of algorithm required for the KBS model building and testing is $O$ (the largest among $2^{m}, R^{2}, S^{2}$ ) where $2^{m}$ is the smallest integer larger than or equal to $N=\operatorname{dim}(\mathbf{v})=\operatorname{dim}\left(\boldsymbol{\nu}^{*}\right), R=\operatorname{dim}\left(\boldsymbol{\nu}_{i}^{*}\right)=\operatorname{dim}\left(\mathbf{v}^{*}\right) \leq N$, and $S=\operatorname{dim}\left(\boldsymbol{\mu}_{i}^{*}\right)=\operatorname{dim}\left(\mathbf{u}^{*}\right) \leq N$. Second, the usability cost is very cheap since the KBS is built by only 20 user's keystroke patterns, $\boldsymbol{\nu}_{i}, i=1, \ldots, 20$, whose size $N$ ranges from 13 to 21 . Third, the practically acceptable classification accuracy is obtained (average FAR $=1.08 \%$, average $\mathrm{FRR}=5.33 \%$ ) at the low cost of usability and computational complexity.

\section{Acknowledgement}

We would like to thank Professor Sungzoon Cho at Seoul National University for sharing his data on keystroke dynamics. This work was supported by grant No.R01-2005-000-103900-0 from the Basic Research Program of the Korea Science and Engineering Foundation.

\section{References}

1. Vidakovic, B.: Statistical Modeling by Wavelets. Wiley (1999)

2. Peacock, A., Ke, X., Wilkerson, M.: Typing Patterns: A Key to User Identification. IEEE Securiy \& Privacy 2 (2004) 40-47

3. Yu, E., Cho, S.: Keystroke dynamics identy verification-its problems and practical solutions. Computers \& Security 23 (2004) 428-440

4. Sheng, Y., Phoha, V.V., Rovnyak, S.M.: A Parallel Decision Tree-Based Method for User Authentication Based on Keystroke Patterns. IEEE Trans. Sytems, Man and Cybernetics, Part B. 35 (2005) 826-833 